\title{
Indicadores de depressão do Zulliger no Sistema Compreensivo (ZSC) ${ }^{1}$
}

\author{
Anna Elisa de Villemor-Amaral ${ }^{2}$ \\ Maria Aparecida dos Santos Machado \\ Universidade São Francisco, Itatiba-SP, Brasil
}

\begin{abstract}
Resumo: Este trabalho teve como objetivo verificar se os indicadores de depressão que compõem a Constelação de Depressão (DEPI) do Rorschach no Sistema Compreensivo (SC) auxiliam no diagnóstico de depressão por meio do Zulliger SC. Participaram 27 pacientes com depressão e 27 não-pacientes, do sexo feminino. Para coleta de dados utilizou-se a SCID-I, questionário de identificação e o Zulliger. O tempo de aplicação dos instrumentos foi de 45 a 100 minutos. Os protocolos foram codificados e recodificados por outra pesquisadora às cegas e eventuais discordâncias foram resolvidas por um terceiro juiz. Após análises estatísticas descritivas, aplicou-se o teste $t$-student. Dentre as variáveis do DEPI, alcançaram valores significativos FD + V, Sum-SH, Índice-egocentricidade, $\mathrm{CF}+\mathrm{C}<\mathrm{FC}$, Determinantes-mistos e Intelectualização. Conclui-se que o ZSC é um instrumento que pode contribuir no diagnóstico da depressão.
\end{abstract}

Palavras-chave: avaliação psicológica, teste de Zulliger, técnicas projetivas, depressão.

\section{The depression index in the Zulliger Comprehensive System (ZSC)}

\begin{abstract}
The aim of this paper is to verify the validation Zulliger in System-Comprehensive (ZSC) in Brazil, considering the indicators of depression of the DEPI Constellation in the Rorschach-SC. The participants were 54 women, 27 patients with depression and 27 non-patients. The instruments were the SCID-I, questionnaire for the identification and Zulliger test. The time of application of the instruments was 45/100 minutes. The protocols were coded and recoded by another researcher blind and any disagreements were resolved by a third judge. After descriptive statistical analysis, was the t-student test. Among the variables of the DEPI, were substantial FD + V, Sum-SH, Index-egocentric, CF + C $<$ FC, mixed-determinants and Intelectualization Concluding, the ZSC is a tool that can help in the diagnosis of depression.
\end{abstract}

Keywords: psychological assessment, Zulliger test, projective techniques, depression.

\section{Indicadores de la depresión en Zulliger en el Sistema Comprehensivo (ZSC)}

Resumen: La finalidad de este trabajo fue verificar la validez de los indicadores de depresión de la constelación DEPI del Rorschach-Sistema Comprehensivo (SC) (considerando el Zulliger SC como instrumento diagnóstico. La muestra incluyó 27 pacientes con depresión y 27 no-pacientes, todas mujeres. Para recoger los datos se utilizó el SCID-I, el cuestionario de identificación y El test de Zulliger. El tiempo de aplicación de los instrumentos varió entre 45 y 100 minutos. Los protocolos fueron codificados y recodificados por otro investigador a ciegas y cualquier desacuerdo se resolvió mediante un tercer juez. Tras el análisis estadístico descriptivo, se hizo la prueba t de Student. Entre las variables estudiadas, alcanzó valores significantes el FD + V, Sum-SH,-índice de egocentrismo, $\mathrm{CF}+\mathrm{C}<\mathrm{FC}$, los determinantes mixto y el índice de intelectualización. Se concluye que el ZSC es una herramienta que puede ayudar en el diagnóstico de la depresión.

Palabras clave: evaluación psicológica, test de Zulliger, técnicas proyectivas, depresión.

A avaliação psicológica é uma atividade desenvolvida no Brasil antes mesmo da Psicologia ser reconhecida como profissão. Próximo à década de 1960, essa atividade foi bastante criticada por vários segmentos sociais porque um grande número de instrumentos utilizados não havia sido submetido a estudos de normatização, validação e precisão para a população brasileira, gerando assim dúvidas sobre a fidedignidade de seus resultados (Pasquali \& Alchieri, 2001).

Nas últimas décadas, com as diretrizes para o estabelecimento de ações técnicas e políticas para a utilização dos testes, a avaliação psicológica reconquista sua importância e status

\footnotetext{
'Apoio: CNPq.

${ }^{2}$ Endereço para correspondência:

Anna Elisa de Villemor-Amaral. R. Alexandre Rodrigues Barbosa, 45. Centro.

CEP 13.251-900. Itatiba-SP, Brasil. E-mail: aevillemor@terra.com.br.
}

na atuação do psicólogo (Noronha \& Alchieri, 2002). Como se trata de uma atividade de grande importância social, pois está presente nos processos de seleção pessoal, na área escolar, jurídica, na clínica propriamente dita e também na pesquisa científica, tem-se enfatizado a importância de desenvolver os instrumentos de modo que atendam aos critérios preconizados pela comunidade científica, tanto no âmbito nacional como internacional.

Os critérios exigidos dizem respeito às qualidades psicométricas de validade, precisão e padronização. Mas, para os métodos de auto-expressão - tradicionalmente chamados métodos projetivos - a adequação a tais critérios não é uma tarefa simples já que seus propósitos e modo de gerar dados a serem interpretados são muito diferentes dos testes que buscam, pelo auto-relato, a quantificação de traços ou de estados.

Os métodos de auto-expressão originaram-se no campo da clínica, no qual a pessoa é observada na relação com o 
observador que procura apreender o maior número possível de informações sobre vários aspectos da dinâmica psíquica, manifestos ou não no comportamento visível, que possibilitarão um diagnóstico e um planejamento de intervenção (Villemor-Amaral, 2006). Isso acarreta diversas dificuldades quando se procura atingir os critérios de excelência no que diz respeito aos atributos psicométricos dos referidos métodos. Entre elas estão a natureza dos fenômenos estudados que nem sempre são observáveis diretamente pelo comportamento; a ocorrência de determinados fenômenos uma única vez em um único indivíduo; a natureza da tarefa que é proposta pelos métodos de auto-expressão ao se apresentar estímulos pouco definidos, podendo gerar uma variedade muito grande de respostas. Além disso, o fato de que em pesquisa, quanto mais complexo o número de variáveis envolvidas na análise, maior deve ser o número de sujeitos estudados é dificilmente praticável em investigações desse tipo, que demandam muito tempo para aplicação e análise dos dados, resultando amostras por vezes mais reduzidas (Güntert, 2001).

Entre os métodos de auto-expressão encontra-se o Teste de Zulliger que examina a estrutura e a dinâmica da personalidade a partir da percepção dos estímulos não estruturados (manchas de tinta) que se apresentam ao sujeito. Esse instrumento foi desenvolvido pelo psicólogo suíço Hans Zulliger, em 1948, visando criar um método com as características fundamentais do Rorschach, mas que possibilitasse avaliar um grande contingente de pessoas, em um menor tempo, de modo rápido e eficaz (Vaz, 2000).

Baseando-se no método de Rorschach, com o qual estava bastante familiarizado, Zulliger elaborou um conjunto com três manchas de tinta impressas em diapositivos para a realização de aplicações coletivas. Posteriormente desenvolveu também uma forma impressa em cartões, visando aplicação individual tal como no Rorschach (Vaz, 2000). Sendo assim, a diferença entre os dois instrumentos é basicamente o número de pranchas, uma vez que o procedimento de classificação das respostas e análise de dados manteve-se igual ao do Rorschach, e a tarefa envolve também a apresentação de estímulos não estruturados que devem ser interpretados, tornando possível obter uma avaliação complexa dos aspectos cognitivos e afetivos da personalidade. No Brasil, o Zulliger se difundiu mais dentro da perspectiva desenvolvida por Bruno Klopfer (1936, citado por Vaz, 2002) para o Rorschach sendo, em nosso meio, adaptado por Vaz (1998) e Freitas (1996). Além disso, poucos autores brasileiros se dedicaram à investigação desse instrumento.

Já o método de Rorschach, ao longo dos anos, sofreu diversas alterações no que diz respeito à aplicação, modo de classificação e interpretação dos dados, na medida em que pesquisas foram realizadas em centros de estudos distantes entre si e conduzidos por autores diferentes. Entretanto, diversas investigações efetuadas por Exner (1995) levaram ao desenvolvimento do Sistema Compreensivo, no intuito de reunir os conhecimentos e as investigações acessíveis naquela época, a partir dos principais sistematizadores deste método nos Estados Unidos (Nascimento \& Güntert, 2000). Samuel Beck, Marguerite
Hertz, Bruno Klopfer, Zigmunt Piotrowski e David Rappaport foram as principais referências de Exner e seu objetivo não era criar um novo sistema, mas superar as complicações do paralelismo dos muitos sistemas existentes que impossibilitavam a troca entre os pesquisadores e impediam o desenvolvimento do Método (Nascimento \& Güntert, 2000).

Exner constatou que muitos pontos relacionados a aspectos importantes da aplicação e da classificação das respostas não dispunham de suficientes investigações sistemáticas e observou que modos de aplicação diferentes resultavam em tipos diferentes de protocolos. Além disso, percebeu que existiam códigos ou critérios de classificação com fraca confirmação empírica ou ainda outros para os quais já existiam pesquisas que traziam resultados negativos a seu respeito. Nessas circunstâncias, o novo sistema passou a integrar os indicadores de qualquer um dos sistemas que pudessem obter uma comprovação empírica satisfatória.

O Sistema Compreensivo continua em constante desenvolvimento, integrando, revisando e acrescentando resultados de investigações extensas, fundamentadas em metodologias atualizadas. Empenhos continuam sendo feitos para aprimorar os critérios da codificação das respostas e para sistematizar as análises dos conteúdos e dos modos de expressão verbal em sua formulação, incluindo contribuições de fundamentação psicanalítica (Nascimento \& Güntert, 2000).

Considerando a semelhança entre as duas técnicas, Zulliger e Rorschach, nas últimas décadas pensou-se na possibilidade de adaptar o primeiro para os mesmos princípios adotados pelo Sistema Compreensivo, a fim de verificar em que medida é possível equiparar os dois métodos quanto ao seu alcance e eficiência. Entretanto, são poucos os estudos relacionados à sistematização do Zulliger para o Sistema Compreensivo. Dentre eles, encontram-se a pesquisa de Zdunic (1999), na Argentina, Brinkmann (1998), no Chile, a de Mattlar, Sandahl, Lindberg e Lehtinen (1990), de Mahmood (1990) na Inglaterra, e Villemor-Amaral e Primi (2009), no Brasil. Entretanto, os estudos encontrados na literatura internacional limitaram-se a fazer uma transposição simples de um sistema para outro e estabelecer padrões normativos, porém não chegaram a se dedicar a pesquisas de validade e de precisão.

Uma das interessantes contribuições do Sistema Compreensivo refere-se à elaboração de constelações de indicadores que, em conjunto, constituem fortes indícios de patologias específicas ou desajustes e que também possibilitam compreender a estrutura da personalidade, estilos ou traços dominantes. Exner criou a Constelação de Suicídio (S-CON); Índice de alteração de pensamento e percepção (PTI); Índice de Déficit Relacional (CDI); Índice de Hipervigilância (HVI); Índice de Estilo Obsessivo (OBS) e, por fim, o Índice de Depressão (DEPI) (Nascimento \& Güntert, 2000).

O DEPI integra 15 variáveis organizadas em sete indicadores que são $(\mathrm{FV}+\mathrm{VF}+\mathrm{V}>0)$ ou $(\mathrm{FD}>2)$; (Det. Mistos de Cor e Sombreado $>0)$ ou $(\mathrm{S}>2) ;(3 \mathrm{r}+(2) / \mathrm{R}>.44 \mathrm{e} \mathrm{Fr}+\mathrm{rF}=$ $0)$ ou $(3 r+(2) / R<.33) ;($ Afr $<.46)$ ou (Det. Misto $<4)$; (soma 
de Sombreado $>$ FM + m) ou (Soma C'>2); $(\mathrm{MOR}>2)$ ou $(2 \mathrm{AB}+\mathrm{Art}+\mathrm{Ay}>3) ;(\mathrm{COP}<2)$ ou (Isolate/R > .24) (Exner, 1999). No Rorschach, a presença de pelo menos cinco desses indicadores revela que o indivíduo apresenta traços encontrados habitualmente em sujeitos diagnosticados com depressão, ou que, por sua organização psicológica, têm facilidade de entrar em estados depressivos, enquanto que a presença de seis ou sete indicadores aponta com maior precisão o transtorno depressivo (Exner, 1993).

Para constituir o DEPI, Exner estudou grupos de distímicos, unipolares, bipolares e esquizoafetivos. Sua conclusão foi de que existem três tipos de sujeitos que são diagnosticados como depressivos ou com um transtorno afetivo, a saber: os que estão emocionalmente alterados; os que se mostram em sua conduta pessimistas, desmotivados e derrotados e os que se sentem desvalidos e inertes para o enfrentamento do cotidiano. Sendo assim, o DEPI é constituído por uma mescla de variáveis relacionadas ao funcionamento cognitivo (MOR, Índice de Egocentrismo, FD e índice de Intelectualização) e ao afetivo (Cor e Sombreado, C', Afr). Para Exner é preciso a conjunção desses tipos de variáveis para o diagnóstico da depressão, pois suas pesquisas evidenciaram que a maioria dos sujeitos diagnosticados como distímicos ou com um transtorno afetivo, têm dificuldades em ambas as áreas (Exner, 1993).

Conforme o Manual diagnóstico e estatístico de transtornos mentais (DSM-IV-TR) (American Psychiatric Association, 2002), a depressão em geral é diagnosticada quando o sujeito apresenta um humor deprimido ou a perda de interesse ou prazer por quase todas as coisas em um período mínimo de quinze dias. Outros sintomas são: alteração do apetite, peso, sono e atividade psicomotora; diminuição de energia; sentimento de desvalia ou culpa; dificuldade para pensar, concentrar-se ou tomar decisões; pensamentos recorrentes sobre morte ou ideação suicida, planos ou tentativas de suicídio. Além do sofrimento, os pacientes têm prejuízos significativos nas funções e relacionamentos sociais (APA, 2002).

Desse modo, o presente estudo teve como objetivo investigar a sensibilidade do Zulliger conforme o Sistema Compreensivo (ZSC) para a depressão, considerando os componentes da constelação DEPI. Adotou-se, como hipótese, que o desempenho de indivíduos com depressão seria diferente de não-pacientes nesses indicadores. Como para o ZSC ainda não estão definidos os valores esperados para esses indicadores, tal como no Rorschach, optou-se, neste estudo, por considerar os valores médios encontrados no grupo de pacientes diagnosticados com depressão, contrapondo-os com os valores médios obtidos no grupo sem essa patologia, procurando-se identificar diferenças significativas entre os dois grupos.

\section{Método}

\section{Participantes}

Participaram deste estudo 54 mulheres, subdivididas em dois grupos. O primeiro grupo foi composto de 27 voluntárias do interior de São Paulo, com idade entre 24 e 57 anos, grau de escolaridade entre ensino fundamental e superior, com diagnóstico de depressão confirmado por meio da SCID-I (Structured Clinical Interview for DSM-IV). As pacientes encontravam-se em tratamento psiquiátrico nos estabelecimentos do SUS (Sistema Único de Saúde) ou em clínicas privadas. Para o grupo de comparação participaram 27 voluntárias de estabelecimentos comerciais, também do interior de São Paulo, equiparadas quanto à idade e à escolaridade com o primeiro grupo. $\mathrm{O}$ critério de inclusão utilizado foi não apresentarem queixas específicas e nunca terem recorrido à ajuda psicológica ou psiquiátrica.

\section{Instrumentos}

Foi utilizada a SCID-I, que se constitui como um roteiro de entrevista que pode ser utilizado por psiquiatras ou outros profissionais da área de Saúde Mental, treinados e familiarizados com a classificação e critérios do DSM-IV. A aplicação completa dos módulos dura aproximadamente uma hora e meia e é dividida em duas etapas. A primeira etapa possibilita a avaliação de aspectos ligados à: identificação; vida escolar e profissional; início, evolução e história do tratamento da enfermidade; contexto ambiental; problemas atuais e funcionamento social na atualidade. A segunda etapa é composta por módulos que avaliam patologias do Eixo I (transtornos afetivos como depressão maior, transtorno somatoforme, esquizofrenia, transtorno obsessivo compulsivo, transtorno do pânico e transtorno do uso do álcool). Para esse estudo, utilizou-se apenas a primeira parte da SCID-I e o módulo concernente aos transtornos afetivos, com o intuito de apenas confirmar o diagnóstico, uma vez que eram pacientes em tratamento para depressão.

Para o grupo de não pacientes, utilizou-se um questionário de identificação composto por perguntas relacionadas à idade, sexo, escolaridade, profissão, humor, ocupação do tempo livre, uso de álcool e droga. A seguir, indagava-se sobre a procura de auxílio profissional para tratamento de problemas psicológicos ou psiquiátricos. Por fim, foi aplicado o teste de Zulliger em todos os participantes.

O Zulliger é um instrumento projetivo composto de três lâminas com manchas de tinta, de 18,5 por $25 \mathrm{~cm}$, sendo uma acromática, uma policromática e outra em vermelho e preto. As manchas são estímulos não estruturados que demandam uma estruturação no ato de se formular alguma resposta que, por sua vez, refletirá aspectos da dinâmica subjetiva do indivíduo. Para sua aplicação o examinador precisa de uma folha de localização que contém uma reprodução reduzida das três pranchas e papel para anotar as respostas, que devem ser registradas exatamente do mesmo modo em que foram emitidas pelo examinando (Villemor-Amaral \& Primi, 2009).

Os critérios de classificação do Sistema Compreensivo são claros e facilmente aplicáveis considerando os seguintes elementos: localização das respostas (W, D, Dd, S); qualidade evolutiva (v, o, + e v/+), que se refere ao nível de integração da resposta; Determinantes, que apontam para as características da mancha que influenciaram a formação das respostas, forma 
(F), movimento (M, FM, m - ativo e passivo), cor (C, CF, FC, $\mathrm{Cn}$ ), cor acromática (C', C'F, FC'), sombreado (Y, YF, FY, V, VF, FV, T, TF, FT), forma e dimensão (FD), pares (2) e reflexos (Fr e rF); qualidade formal (+, o, u, -), que indica o grau de precisão e objetividade da percepção; conteúdo, composto por 26 categorias que agrupam os conteúdos das respostas tais como Humano (H), Animal (A), entre outros; Respostas Populares (P) que são aquelas de alta frequência na população; nota $Z$, que representa a atividade organizativa da percepção, levando-se em conta as qualidades gestálticas diferentes de cada mancha. Finalmente são atribuídos Códigos Especiais para verbalizações pouco comuns feitas pelos indivíduos ao dar suas respostas. São eles (DV, DR, INCOM, FABCOM, CONTAM, ALOG, PSV, CONFAB, AB, AG, COP e MOR, PER e $\mathrm{CP})$. Todos os itens de classificação das respostas, bem como fórmulas e proporções extraídas destes, são organizados no sumário estrutural para a interpretação do protocolo.

O sumário estrutural é dividido em duas partes: a seção superior, para anotar a frequência de cada um dos códigos, e a inferior, para o registro das proporções, razões, porcentagens ou derivações. Na seção inferior, os dados são agrupados em blocos, nos quais os elementos são relativos a uma esfera da personalidade. Sendo assim, têm-se os agrupamentos que informam sobre Controle e Tolerância ao Estresse; ideação; mediação; processamento; afeto; relacionamento interpessoal e autopercepção (Exner, 1999).

\section{Considerações éticas}

Os participantes foram esclarecidos quanto aos objetivos da pesquisa e aos cuidados éticos a serem tomados em relação ao sigilo de suas identidades. Após o rapport, lia-se e assinavase o Termo de Consentimentos Livre e Esclarecido.

\section{Procedimentos}

\section{Coleta de dados}

Foram realizados contatos com os responsáveis pelos estabelecimentos do SUS e das clínicas particulares de uma cidade do interior São Paulo, para a coleta dos dados do grupo de pacientes. Para o grupo de comparação, foram contatados gerentes de estabelecimentos comerciais, também de uma cidade do interior São Paulo, para disponibilizar funcionários para a pesquisa.

O contato com cada voluntária foi individual. No grupo de pacientes, após o rapport e assinatura do Termo de Consentimento Livre e Esclarecido, aplicou-se, em um tempo aproximado de uma hora, a SCID-I, módulo de Avaliação preliminar - revisão geral (dados demográficos, história escolar e ocupacional, estado do tratamento atual, queixa principal e descrição do problema atual, início ou exacerbação da doença atual, novos sintomas ou recorrência, contexto ambiental e possíveis fatores precipitantes, curso ou exacerbação da doença atual, história de tratamento, outros problemas atuais, funcionamento social atual) e o módulo que avalia Episódio Depressivo Maior, com a finalidade de confirmar a patologia em questão. Isso porque a estrutura modular da SCID permite que se façam adaptações de acordo com as situações e contextos de aplicação. Tavares (2000) destaca que em pesquisas, quando o foco está em transtornos específicos, é possível aplicar apenas o módulo que avalia a patologia que se pretende investigar.

Em seguida aplicou-se o Zulliger em um tempo aproximado de 30 minutos. Sua aplicação consistiu em apresentar as pranchas, uma a uma, perguntando: 'Com que ela se parece?'. Após a apresentação das três pranchas, iniciou-se o inquérito, reapresentando as figuras na mesma sequência, repetindo o que foi dito e perguntando o local em que o sujeito viu a resposta (marcando-se na folha de localização) e o quê na prancha the sugeriu essa idéia. As respostas foram registradas na íntegra tal como foram pronunciadas, anotando-se também as atitudes e expressões do sujeito ao formular suas respostas.

No grupo de não-pacientes, após o rapport e assinatura do Termo de Consentimento Livre e Esclarecido, aplicou-se o questionário para levantamento dos dados demográficos e verificação dos critérios de inclusão, seguido da aplicação do Zulliger. Optou-se por considerar a ausência de queixas específicas e de busca por tratamento psicológico ou psiquiátrico, seguindo com isso o mesmo critério adotado por Exner (1995) em suas pesquisas. A aplicação dos instrumentos também foi individual, em um tempo total aproximado de 45 minutos.

\section{Análise dos dados}

As respostas dadas ao Zulliger foram codificadas conforme os critérios do Sistema Compreensivo e, para confiabilidade dos dados, os protocolos foram recodificados por um juiz independente. Quando havia discordância os índices foram corrigidos com auxílio de um terceiro juiz. Para atingir o objetivo deste estudo, consideraram-se os 15 indicadores que compõem a constelação DEPI, que são: ( FV $+\mathrm{VF}+\mathrm{V}>0)$ ou $(\mathrm{FD}>2)$; (Det. Mistos de Cor e Sombreado $>0)$ ou $(\mathrm{S}>2) ;(3 \mathrm{r}+(2) / \mathrm{R}$ $>.44 \mathrm{e} \mathrm{Fr}+\mathrm{rF}=0)$ ou $(3 \mathrm{r}+(2) / \mathrm{R}<.33) ;(\mathrm{Afr}<.46)$ ou (Det. Misto $<4)$; (soma de Sombreado $>$ FM + m) ou (Soma C'> 2); $(\mathrm{MOR}>2)$ ou $(2 \mathrm{AB}+\mathrm{Art}+\mathrm{Ay}>3) ;(\mathrm{COP}<2)$ ou $($ Isolate/ $\mathrm{R}$ $>$.24) (Exner, 1999).

Esses indicadores constituem uma mescla de variáveis que combinam aspectos do funcionamento cognitivo e afetivo, incluindo índices da relação interpessoal. $O$ código especial MOR, Índice de Intelectualização (2AB+Art+Ay), FD e Índice de Egocentricidade $(3 r+(2) / R)$, estão relacionados a autopercepção e ideação. Já os VF, FV, V, C', Afr, Det. Misto, S, Det. Misto de cor e sombreado referem-se à afetividade. Aspectos interpessoais são observados com o código especial COP e o Índice de Isolamento (Isolate/R).

Após tabulação dos dados dos protocolos em planilhas Excel, foram calculadas médias e desvios padrão dos códigos e indicadores que compõem a constelação DEPI, realizando-se em seguida o teste $t$-student para comparar o desempenho entre os grupos nessas variáveis. 


\section{Resultados e Discussão}

A avaliação psicológica é uma atividade que visa contribuir para uma melhor compreensão do indivíduo, seus recursos e suas dificuldades adapatativas, disponibilizando informações que podem auxiliar no planejamento de estratégias de intervenção para os fenômenos psicológicos, coletivos ou individuais. Essa atividade é o resultado de um processo que envolve muitas variáveis, entre as quais, a formação do profissional habilitado para executá-la e a qualidade dos instrumentos utilizados são imprescindíveis. Por isso a comunidade científica estabeleceu diretrizes de ações técnicas e políticas para a utilização dos testes psicológicos (Noronha \& Alchieri, 2002).
A qualidade dos instrumentos de avaliação psicológica está atrelada a sua validade, precisão e padronização. Isso é um grande desafio para os métodos de auto-expressão já que seus objetivos não se restringem a medir uma única variável e, sim, um conjunto de aspectos que possibilitam compreender a dinâmica subjetiva do sujeito, sua singularidade (VillemorAmaral, 2006).

O presente estudo buscou verificar a sensibilidade do ZSC para o diagnóstico da depressão, utilizando como parâmetro a constelação DEPI do Sistema Compreensivo do Rorschach. Todas as variáveis que compõem o Índice de Depressão foram submetidas às análises estatísticas e serão discutidas aquelas cujos resultados foram significativos do ponto de vista estatístico. A Tabela 1 apresenta os resultados obtidos.

Tabela 1

Média, desvio padrão e teste t-student de variáveis que compõem o DEPI

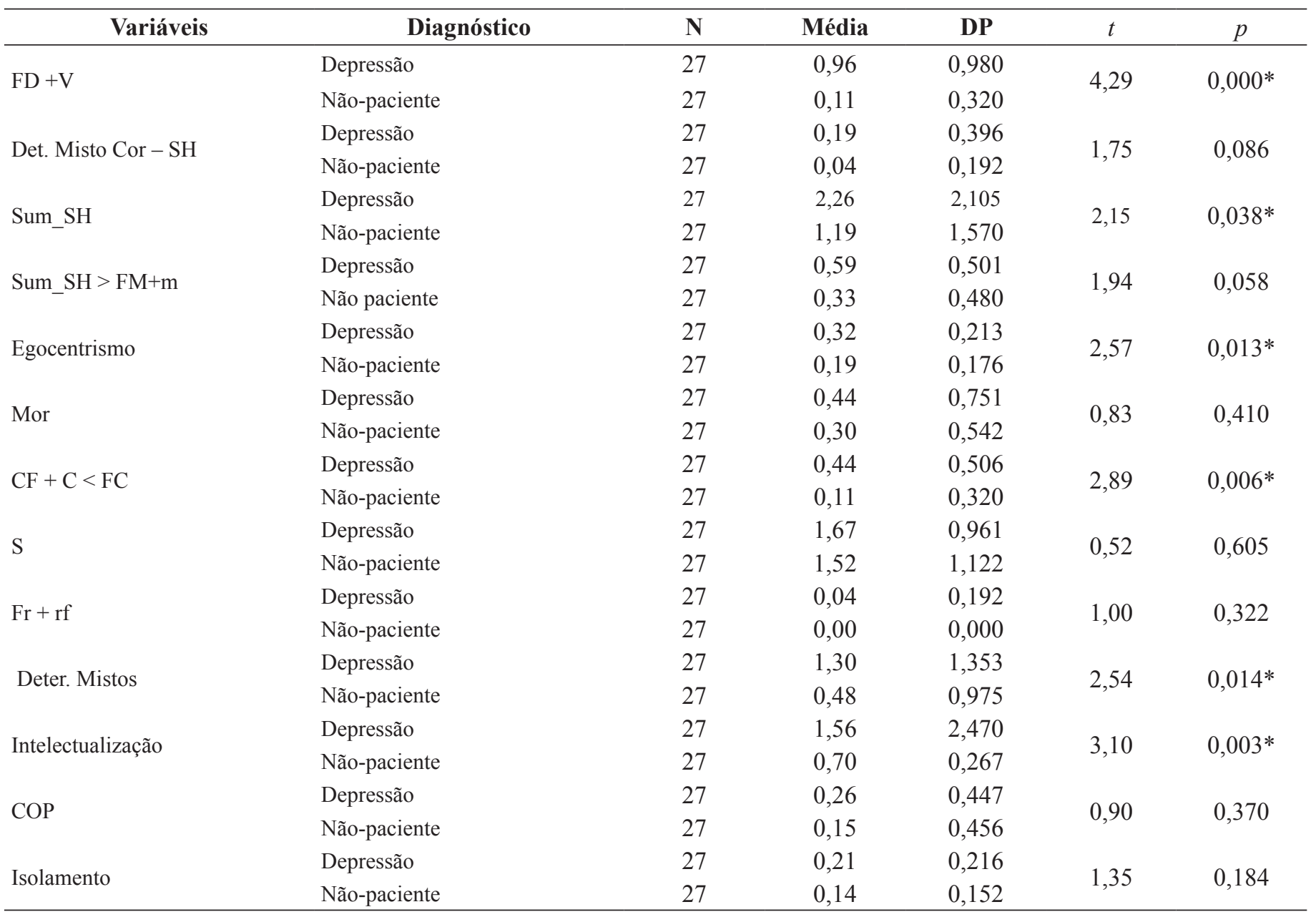

$* p<0,05$.

A partir da Tabela 1, nota-se que os indicadores FD + V, Sum_SH, Egocentrismo, CF $+\mathrm{C}<\mathrm{FC}$, Determinantes mistos e Intelectualização alcançaram valores significativamente aumentados no grupo de pacientes com depressão. Por sua vez, os indicadores Determinantes Misto Cor-SH e Sum_SH > FM $+\mathrm{m}$ alcançaram uma diferença de média com valor de $\mathrm{p}=0,086$ e $p=0,058$, respectivamente, ou seja, tratam-se de resultados marginalmente significativos. 
Os indicadores FD e V, que aparecem aumentados do grupo de depressivos, estão relacionados ao processo de autoinspeção, por isso são tão importantes quando se fala da autoimagem do indivíduo. O V mais especificamente representa o uso da introspecção com matizes de autocrítica negativa e, por isso, é sinal de baixa auto-estima. Já o FD representa o uso da introspecção e a tomada de distância com o meio, sem acrescentar o tom de autocrítica negativo das respostas V. Porém, quando o número de FD é elevado, significa que o indivíduo dedica muito esforço às tarefas de auto-inspeção, podendo ocasionar isolamento pessoal em relação a seu meio de convívio.

O Índice de Egocentrismo constitui uma medida da proporção em que o indivíduo volta sua atenção para si e para os outros. Esse indicador está aumentado no grupo de depressivos, indicando um alto nível de atenção voltada para si mesmo. Caracteriza motivação do indivíduo para se auto-observar, o que, associado ao aumento de FD+V e Determinantes mistos, reforça o caráter negativo de ruminações depressivas a respeito de si mesmo.

Os indicadores FC, CF, C estão relacionados com a percepção da forma e cor dos estímulos, sendo que, respectivamente, FC indicaria experiências afetivas controladas ou direcionadas por elementos cognitivos, enquanto $\mathrm{CF}$ e $\mathrm{C}$ estariam relacionados a descargas afetivas com pouca ou nenhuma modulação cognitiva. Adultos costumam apresentar $\mathrm{FC}>\mathrm{CF}+\mathrm{C}$, porém os pacientes psiquiátricos têm problemas no controle emocional, sendo exceção os psicossomáticos que geralmente apresentam $\mathrm{FC}$ exageradamente maior que $\mathrm{CF}+\mathrm{C}$ ou ausência de resposta de cor e presença de resposta do grupo C' (Villemor-Amaral \& Primi, 2009). No grupo de pacientes com depressão, a frequência de $\mathrm{CF}+\mathrm{C}$ foi menor que $\mathrm{FC}$, sendo significativa a diferença com o grupo de não paciente $(p=0,006)$, mostrando que existe um controle maior, uma ausência de espontaneidade dos afetos no grupo de pacientes. É interessante destacar que na população de não pacientes, é esperado que $\mathrm{FC}$ seja maior que a soma de CF e C mas a predominância absoluta de FC deixa de ser favorável pois indica pouca expontaneidade na expressão emocional, o que se aplica aos pacientes da amostra em estudo.

A presença de Determinantes Mistos no protocolo indica maior envolvimento do sujeito com a tarefa proposta. Esse foi mais um indicador com diferença significativa para o grupo de pacientes. Outro indicador aumentado e de valor significativo no grupo de depressivos foi o Índice de Intelectualização. Um protocolo que apresente alto índice de Intelectualização sugere indivíduo com tendência a neutralizar o efeito das emoções, utilizando os processamentos cognitivos. Trata-se de mecanismo pseudo-intelectual para tentar fugir ou desmentir a presença do afeto e, sobretudo, suas consequências disfóricas. Com isso, suavizam-se os seus efeitos, mas também se reduz a possibilidade de manejar as emoções de modo direto e realista. Nestes casos, os indivíduos tendem a ficar vulneráveis à desorganização em situações de sobrecarga emocional, porque esta estratégia ideativa perde a eficácia quando há um aumento da intensidade dos estímulos afetivos.
Em nossa amostra, os indicadores Mor, S, Fr+rF, COP e Isolamento, não mostraram diferenças estatísticas significativas. Por sua vez, os indicadores Sum $\mathrm{SH}>\mathrm{FM}+\mathrm{m}$ e CSH alcançaram valores marginalmente significativos.

A presença das respostas Cor-sombreado (CSH) tem importância especial para o estudo dos aspectos afetivos. De acordo com Exner e Sendín (1999), as respostas CSH são pouco comuns e só são observadas, praticamente, em protocolos de depressivos. A autora afirma a importância de analisar se o componente do sombreado é Y ou C', V ou T. Como nesta pesquisa, observou-se também um aumento de $\mathrm{V}$, que são determinantes mais estáveis em caso de teste e reteste, conclui-se que o aumento do sombreado está bastante atrelado a esta variável e por isso CSH reflete também um fenômeno psíquico mais crônico, refletindo uma ambivalência de tipo caracterial que faz com que o indivíduo confunda as emoções e tenha sentimentos positivos e negativos na mesma situação. Estes indivíduos costumam experenciar emoção mais intensa do que a maioria e, com frequência, lhes é difícil conter ou terminar uma situação afetiva. A ambivalência, quando é um traço caracterial, supõe uma grande dificuldade para manter a coerência das reações emocionais nos diversos tipos de estimulação afetiva e pode afetar muito negativamente as relações desse indivíduo com o meio.

O Índice de Isolamento apresentou maior média no grupo de depressivos, mesmo que não tenha alcançado diferença significativa do grupo de não pacientes. Isto pode apontar para uma tendência, entre os pacientes, de se afastarem do ambiente social, já que esse índice aparece em sujeitos menos envolvidos nas interações pessoais. Isso não significa, necessariamente, que tenham desadaptações ou conflitos manifestos, mas que apresentam maior retraimento nos contatos ou intercâmbios com o meio externo.

\section{Considerações finais}

Para concluir, os resultados demonstram que os indicadores de depressão no Rorschach-SC podem ser úteis para a avaliação dessa patologia por meio do Zulliger, quando codificado e analisado na perspectiva do Sistema Compreensivo. Sendo assim, o ZSC permite auxiliar com o diagnóstico de depressão, considerando-se os indicadores usados no Rorschach. De certa forma, os resultados favoráveis nesse estudo contribuem também, de modo mais amplo, com evidências de validade para o uso do Zulliger-SC em nosso meio. Entretanto deve-se destacar que esse estudo restringiu-se a uma amostra pequena, exclusivamente do sexo feminino e certamente somente estudos com amostras maiores e mais diversificadas poderão conferir maior segurança aos resultados. Outros estudos que busquem evidências de validade para essa técnica diagnóstica ainda precisam ser feitos, apesar de já se contar com vários dados favoráveis nesse sentido.

Um problema fundamental ainda a ser investigado referese à possibilidade de encontrar notas de corte para cada um 
dos indicadores do Zulliger componentes da Constelação de Depressão, assim como de outras constelações de sinais patológicos, tal como previsto no Rorschach-SC. Sendo assim, os futuros estudos deverão voltar-se também para a definição de valores limite que ajudem a diferenciar indivíduos com ou sem patologia em cada componente da DEPI ou outras patologias não abordadas nesse estudo.

De qualquer modo, é importante lembrar que diagnósticos nosográficos conforme os critérios dos manuais de classificação das doenças não são plenamente definidos por meio das diversas técnicas projetivas, incluindo-se o Rorschach. Estudos com grupos patológicos podem ser úteis para demonstrar algumas evidencias de validade, mas o que se espera do Zulliger e de outros Métodos Projetivos é que contribuam de modo confiável na compreensão de dinamismos da personalidade, principalmente associando-os a um conjunto de outras estratégias em um contexto de avaliação.

\section{Referências}

American Psychiatric Association (2002). Manual diagnóstico e estatístico de transtornos mentais DSM-IV-TR (C. Dornelles, Trad.). Porto Alegre: ArtMed.

Brinkmann, H. (1998). Proposición de parâmetros para el Test de Zulliger (Z). Revista Chilena de Psicología, 19(2), $5-47$.

Exner, J.E., Jr. (1993). ElRorschach: Un sistema comprehensivo. Vol. 1: Fundamentos Básicos (3a ed.). Madrid: Psimática.

Exner, J. E., Jr. (1995). Issues and methods in Rorschach research. New Jersey: Lawrence Erlbaum Associates Publishers.

Exner, J. E., Jr. (1999). Manual de classificação do Rorschach para o sistema compreensivo. São Paulo: Casa do Psicólogo.

Exner, J. E., Jr., \& Sendín, C. (1999). Manual de interpretação do Rorschach para o sistema compreensivo. São Paulo: Casa do Psicólogo.

Freitas, A. M. L. (1996). Teste Zulliger: Aplicação e avaliação. São Paulo: Casa do Psicólogo.

Güntert, A. E. V. A. (2001). Técnicas projetivas: O geral e o singular em avaliação psicológica. In F. Sisto, E. T. B. Sbardelini, \& R. Primi, Contextos e questões em avaliação psicológica (pp. 77-85). São Paulo: Casa do Psicólogo.

Mahmood, Z. (1990). The Zulliger Test. British Journal of Projective Psychology, 35(2), 2-16.

Mattlar, C. E., Sandahl, C., Lindberg, S., \& Lehtinen, V. (1990). Methodological issues associated with the application of the comprehensive system when analyzing the Zulliger, and the structural resemblance between the Zulliger and the Rorschach. Bristish Journal of Projective Psychology, 35(2), 17-27.

Nascimento, R. S. G. F., \& Güntert, A. E. V. A. (2000). Novas tendências: Introdução ao Sistema Compreensivo de Exner. In J. A. Cunha, Psicodiagnóstico-V (5a ed., pp. 368-377). Porto Alegre: ArtMed.
Noronha, A. P. P., \& Alchieri, J. C. (2002). Reflexões sobre os instrumentos de avaliação psicológica. In: R. Primi (Org.), Temas em avaliação psicológica (pp. 7-15). São Paulo: Impressão Digital do Brasil.

Pasquali, L., \& Alchieri, L. C. (2001). Os testes psicológicos no Brasil. In L. Pasquali (Org.), Técnica de Exame PsicológicoTEP: Manual (pp. 195-221). São Paulo: Casa do Psicólogo, Conselho Federal de Psicologia.

Tavares, M. (2000). A entrevista estruturada para o DSM-IV. In J. A. Cunha, Psicodiagnóstico-V (5a ed., pp. 75-87). Porto Alegre: ArtMed.

Vaz, C. E. (1998). Zulliger: A técnica de Zulliger forma coletiva. São Paulo: Casa do Psicólogo.

Vaz, C. E. (2000). A técnica de Zulliger no processo de avaliação da personalidade. In J. A. Cunha, Psicodiagnóstico-V (5a ed., pp. 386-387). Porto Alegre: ArtMed.

Vaz, C. E. (2002). Z-Teste: Técnica de Zulliger: Forma coletiva (2a ed.). São Paulo: Casa do Psicólogo.

Villemor-Amaral, A. E. (2006). Desafios para a cientificidade das técnicas projetivas. In A. P. P. Noronha \& F. F. Sisto (Orgs.), Facetas do fazer em avaliação psicológica (pp. 163-171). São Paulo: Vetor.

Villemor-Amaral, A. E., \& Primi, R. (2009). Manual de codificação e interpretação do Zulliger no sistema compreensivo. São Paulo: Casa do Psicólogo.

Zdunic, A. L. (1999). El Teste de Zulliger en la evaluación de personal: Aportes del Sistema Comprehensivo de Exner. Argentina: Paidós.

Anna Elisa de Villemor-Amaral é Professora Associada do Programa de Pós-graduação Stricto Sensu em Psicologia da Universidade São Francisco, campus Itatiba.

Maria Aparecida Santos Machado é psicóloga graduada pela Universidade São Francisco, bolsista PROBAIC.

Recebido: 07/08/2009

$1^{a}$ revisão: $18 / 04 / 2010$

$2^{a}$ revisão: 25/08/2010

Aceite final: 29/09/2010 